\title{
Chemical recycling of polyhydroxyalkanoates as a method towards sustainable development.
}

\begin{abstract}
Chemical recycling of bio-based polymers polyhydroxyalkanoates (PHAs) by thermal degradation was investigated from the viewpoint of biorefinery. The thermal degradation resulted in successful transformation of PHAs into vinyl monomers using alkali earth compound (AEC) catalysts. Poly(3-hydroxybutyrate-co-3-hydroxyvalerate)s (PHBVs) were smoothly and selectively depolymerized into crotonic (CA) and 2-pentenoic (2-PA) acids at lower degradation temperatures in the presence of $\mathrm{CaO}$ and $\mathrm{Mg}(\mathrm{OH})(2)$ as catalysts. Obtained CA from 3-hydroxybutyrate sequences in PHBV was copolymerized with acrylic acid to produce useful water-soluble copolymers, poly(crotonic acid-co-acrylic acid) that have high glass-transition temperatures. The copolymerization of CA derived from PHA pyrolysis is an example of cascade utilization of PHAs, which meets the idea of sustainable development.
\end{abstract}

Keyword: Biopolymers; Chemical recycling; Crotonic acid; Polyhydroxyalkanoates; Thermal degradation. 